\title{
Relationship between the triglyceride glucose index and collateral index in patients with coronary chronic total occlusion
}

\author{
Cihan Aydın' 10 , Halil Berkan Özpak²® \\ ${ }^{1}$ Department of Cardiology, Namık Kemal University, Faculty of Medicine, Tekirdağ, Turkey \\ ${ }^{2}$ Department of Cardiovascular Surgery, Bandırma Training and Research Hospital, Balıkesir, Turkey \\ Received: October 20, 2021 Accepted: October 23, 2021 Published online: November 12, 2021
}

\section{ABSTRACT}

Objectives: This study aims to investigate the relationship between the triglyceride glucose (TyG) index and coronary collateral circulation (CCC) in patients with coronary chronic total occlusion (CTO).

Patients and methods: Between July 2018 and December 2019, a total of 228 consecutive patients (186 males, 42 females; mean age: $62.2 \pm 9.7$ years; range, 18 to 80 years) with stable or unstable angina pectoris who had CCO in at least one coronary artery were retrospectively analyzed. The TyG index was calculated. Coronary collateral circulation was evaluated using the Rentrop grading system. The patients were divided into two groups as low-grade CCC (Group 1, n=101) and high-grade CCC (Group 2, n=127).

Results: There was no significant difference in the body mass index, left ventricular ejection fraction, height, weight, the frequency of dyslipidemia, hypertension, diabetes mellitus, and smoking between the groups ( $p>0.01)$. In the multivariate logistic regression analysis, high TyG index (odds ratio [OR]: 1.345; 95\% confidence interval [CI]: 1.120-2.184; $\mathrm{p}<0.001$ ) and uric acid levels (OR: 0.249; 95\% CI: $0.105-0.491 ; \mathrm{p}=0.013)$ were the independent predictors of poor CCC.

Conclusion: Our study results suggest that a high TyG index is related to poor collateral circulation.

Keywords: Atherosclerosis, coronary collateral circulation, coronary occlusion, dyslipidemia, glucose.

Cardiovascular diseases are among the leading causes of mortality and morbidity worldwide. ${ }^{[1]}$ Coronary chronic total occlusion (CTO) is a condition in which the coronary artery is completely occluded for at least three months and there is no blood flow through the occluded vessel. ${ }^{[2]}$ It is approximately detected in $20 \%$ of patients with coronary artery disease (CAD) ${ }^{[3]}$ When the blood flow decreases due to stenosis in a coronary artery, the collateral vessels progressively open and begin to transport blood to the ischemic or infracted myocardium.

The presence of coronary collateral circulation (CCC) is important for the prevention of ventricular dysfunction and ventricular aneurysm formation. The mechanism of CCC formation has not been clarified, yet. Vascular growth factors and blood cells such as monocytes, neutrophils, and lymphocytes are implicated in the CCC formation. ${ }^{[4]} \mathrm{A}$ good CCC is important for the prognosis of the patient in the long-term. The triglyceride glucose (TyG) index is considered a basic indicator of insulin resistance which can be important for predicting CCC grade. The TyG index, a consequence of triglycerides and fasting plasma glucose (FPG), is a good sign of insulin resistance. ${ }^{[5]}$

The TyG index is significantly related to a raised risk of developing type 2 diabetes mellitus, arterial stiffness, coronary artery calcification, hypertension, and adverse cardiovascular events. ${ }^{[6-9]}$ In many studies, the relationship between the TyG index and atherosclerosis has been proven. ${ }^{[10,11]}$ Alizargar and $\mathrm{Bai}^{[12]}$ reported that the TyG index was significantly related to the total amount of carotid plaque and the increased intima-media thicknesses of carotid arteries in hypertensive and normotensive patients.

In previous studies, higher $\mathrm{TyG}$ index levels were related to poor prognosis in patients with acute

Corresponding author: Cihan Aydın, MD. Namık Kemal Üniversitesi Tıp Fakültesi Kardiyoloji Anabilim Dal, 59030 Tekirdağ, Türkiye.

Tel: +90 506 - 3076616 e-mail: drcihanaydin@hotmail.com

Citation:

Aydın C, Özpak HB. Relationship between the triglyceride glucose index and collateral index in patients with coronary chronic total occlusion. Cardiovasc Surg Int 2021;8(3):154-161. 
ST-elevation myocardial infarction. ${ }^{[13]}$ However, the relationship between $\mathrm{CCC}$ and TyG index in patients with CTO has not been studied in any study to date. In this study, we aimed to investigate the relationship between the TyG index and $\mathrm{CCC}$ in patients with CTO.

\section{PATIENTS AND METHODS}

This single-center, retrospective study was conducted at Namık Kemal University Health Practice and Research Hospital, Department of Cardiology between July 2018 and December 2019. Our center is a respected health center in the Thrace region where 4,000 coronary angiography procedures are performed annually. Angiography was performed in 3,800 patients in our clinic and CTO was detected in 290 patients. Patients with severe renal insufficiency (creatinine $>2 \mathrm{mg} / \mathrm{dL}$ ), elevated triglyceride levels $(\geq 400 \mathrm{mg} / \mathrm{dL})$, active infection, and malignancy were excluded from the study. Patients taking triglyceridelowering medications were also excluded. Finally, a total of 228 consecutive patients (186 males, 42 females; mean age: $62.2 \pm 9.7$ years; range, 18 to 80 years) with stable or unstable angina pectoris were included. Baseline demographic and clinical characteristics of the patients were obtained from the hospital database. A written informed consent was obtained from each patient. The study protocol was approved by the Namık Kemal University, Faculty of Medicine Ethics Committee (Date/No: 25/07/2021/2021,242,10,06). The study was conducted in accordance with the principles of the Declaration of Helsinki.

The fasting blood samples were obtained from all patients during hospitalization after $12 \mathrm{~h}$ of fasting. We performed a complete blood count with an automatic blood analyzer and biochemical values were measured with an automatic device via the standard laboratory techniques.

Blood pressure (BP) was measured three times using an automatic BP monitor on both arms, with the arm placed at heart level after a 10 -min rest period, and an average of three measurements were taken. Patients with an average of these three measurements $>140 / 90 \mathrm{mmHg}$ or those taking antihypertensive drugs were considered hypertensive. Patients with an FPG level of $\geq 7.0 \mathrm{mmol} / \mathrm{L}(126 \mathrm{mg} / \mathrm{dL})$ or hemoglobin $\mathrm{A} 1 \mathrm{c}(\mathrm{HbA} 1 \mathrm{c})$ of $\geq 6.5 \%$ or using antidiabetic medication were defined as diabetic. Hyperlipidemia was defined as being on lipid-lowering therapy or having a total cholesterol level above $220 \mathrm{mg} / \mathrm{dL}$. The TyG was calculated as follows: [fasting triglycerides $(\mathrm{mg} / \mathrm{dL}) \times$ fasting glucose $(\mathrm{mg} / \mathrm{dL}) / 2]{ }^{[14]}$

\section{Coronary angiography}

Coronary angiography images of the patients were evaluated by two independent invasive cardiologists who were blinded to clinical data of the patients. Stenosis of $\geq 50 \%$ more in the coronary arteries was considered significant. Each coronary artery was visualized in at least two different projections. The CCC was evaluated according to the Rentrop classification as described previously. The Rentrop classification was as follows: Grade $0=$ no visible collateral, Grade 1= filling in collateral via collateral vessels without visualizing the side branch epicardial segment, Grade 2= epicardial partial filling coronary artery, and Grade 3= complete filling of the epicardial coronary artery. ${ }^{[15]}$ The patients whose coronary angiography reports were examined were divided into two groups under the Rentrop classification: Group 1 (Grade 0-1) and Group 2 (Grade 2-3). These groups were compared in terms of the calculated $\mathrm{TyG}$ index and other biochemical parameters.

\section{Statistical analysis}

Statistical analysis was performed using the IBM SPSS version 22.0 software (IBM Corp., Armonk, NY, USA). Continuous variables were presented in mean \pm standard deviation (SD) or median (min-max), while categorical variables were presented in number and frequency. The variables were analyzed using the chi-square or Fischer exact test. The distribution of the data was checked using the Kolmogorov-Smirnov test. The Student t-test was used for continuous data conforming to the normal distribution. Non-parametric variables were analyzed using the Mann-Whitney $\mathrm{U}$ test. Receiver operating characteristic (ROC) analysis was performed to determine the optimal cut-off value of the TyG index in the prediction of CCC. The correlation analysis between the TyG index and the CCC was performed using the Spearman correlation test. Multivariate logistic regression analysis was used to identify independent predictors of the CCC. A $p$ value of $<0.05$ was considered statistically significant. coronary collateral index $(\mathrm{CCI})$.

\section{RESULTS}

Baseline characteristics and laboratory results of the patients are summarized in Tables 1 and 2, 


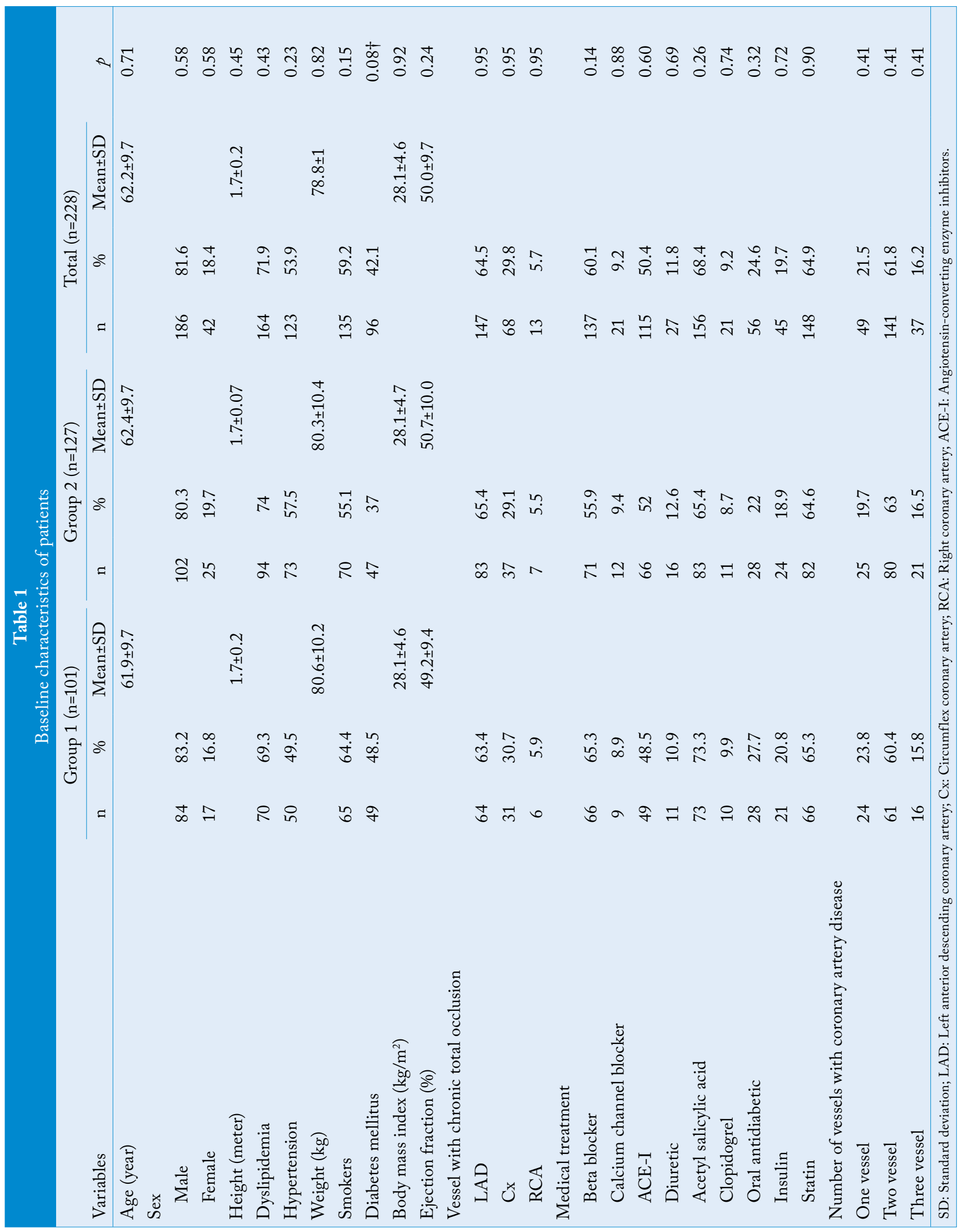




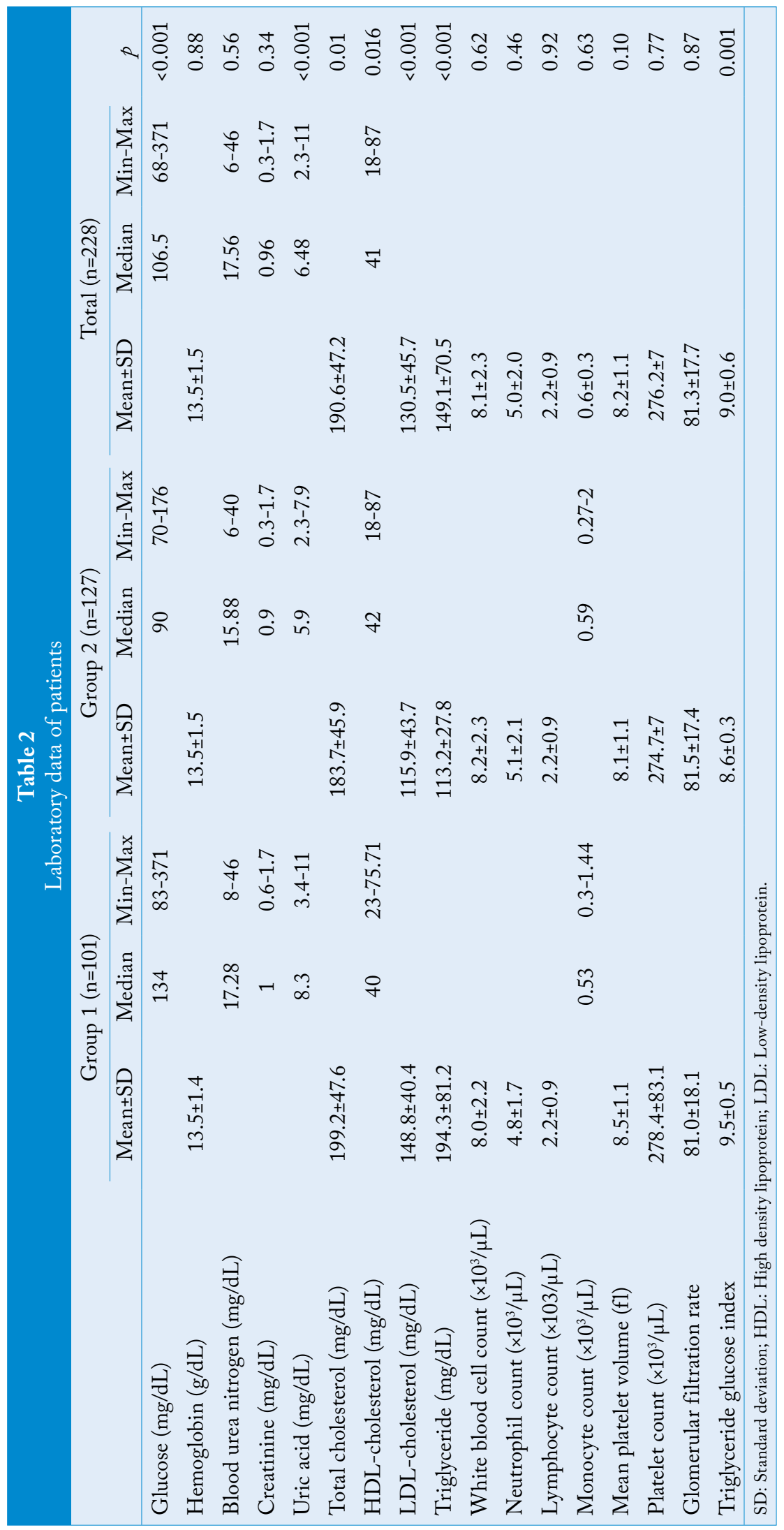




\begin{tabular}{|lccc|}
\hline \multicolumn{4}{|c|}{ Table 3 } \\
Multivariate logistic regression analysis of variables related to CCC \\
\hline Variables & Odds ratio & $95 \%$ CI & $p$ \\
\hline Total cholesterol (mg/dL) & 1.037 & $0.929-1.157$ & 0.517 \\
LDL-cholesterol (mg/dL) & 0.874 & $0.753-1.194$ & 0.077 \\
Triglyceride glucose index & 1.345 & $1.120-2.184$ & $<0.001$ \\
HDL-cholesterol (mg/dL) & 1.060 & $0.880-1.278$ & 0.539 \\
Uric acid (mg/dL) & 0.249 & $0.105-0.491$ & 0.013 \\
\hline CCC: Coronary collateral circulation; CI: Confidence interval; LDL: Low-density lipoprotein; HDL: High density \\
lipoprotein. & & \\
\hline
\end{tabular}

respectively. Of a total of 228 patients, Group 1 consisted of 101 patients (mean age: $63.9 \pm 9.9$ years) and Group 2 consisted of 127 patients (mean age: $62.1 \pm 9.4$ years $)(p=0.710)$. The body mass index, left ventricular ejection fraction, height, and weight of the patients were similar in both groups. The frequency of dyslipidemia, hypertension, diabetes mellitus, and smoking were also similar between the groups. The vessels with chronic total occlusion, the number of vessels with $\mathrm{CAD}$, and the medical treatment of both groups were similar.

Furthermore, the laboratory parameters of the two groups were similar, except for the glucose, uric acid, triglyceride, TyG index, and lipid parameters. However, uric acid $(\mathrm{p}<0.001)$, glucose $(\mathrm{p}<0.001)$,

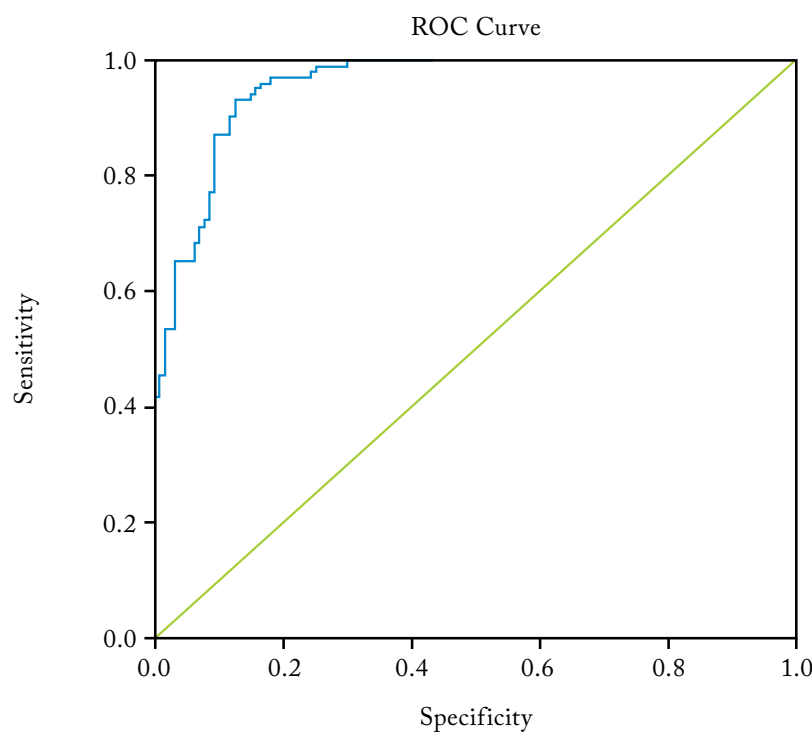

Figure 1. Receiver operating characteristic curve analysis of the triglyceride glucose index levels for the prediction of coronary collateral circulation grade.

ROC: Receiver operating characteristic. triglyceride $(\mathrm{p}<0.001)$, low-density lipoprotein cholesterol $(\mathrm{p}<0.001), \mathrm{TyG}$ index levels $(\mathrm{p}<0.001)$, and total cholesterol $(\mathrm{p}=0.01)$ were higher in Group 1 than Group 2. High-density lipoprotein cholesterol was lower in Group 1 than Group 2 ( $\mathrm{p}=0.016)$.

In the multivariate logistic regression analysis, high TyG index (odds ratio [OR]: 1.345; 95\% confidence interval [CI]: 1.120- 2.184; $\mathrm{p}<0.001)$ and uric acid levels (OR: 0.249; 95\% CI: 0.105-0.491; $\mathrm{p}=0.013$ ) were the independent predictors of poor CCC.

The result of the ROC analysis and area under the curve (AUC) for the TyG index to predict low degree of CCC were as follows: cut-off 8.93, AUC: 0.955, 95\% CI: $0.931-0.978, \mathrm{p}<0.001$ with $88.1 \%$ sensitivity and $88.2 \%$ specificity (Figure 1 ).

In the correlation analysis, a high degree of negative correlation was observed between the TyG index and the CCI $(r=-0.782, p<0.001)$.

\section{DISCUSSION}

In our study, we examined the relationship between the coronary collateral index and TyG index in patients with stable or unstable angina pectoris who underwent coronary angiography procedures. To the best of our knowledge, there is no study regarding the $\mathrm{TyG}$ index and CTO. Early identification of diabetic patients with acute coronary syndrome (ACS) is important to reduce future cardiovascular events. Insulin resistance is an increasingly common metabolic disorder caused by an impaired physiological response to insulin. For many years, insulin resistance and hypertriglyceridemia have been related to metabolic disorders, type 2 diabetes, and atherosclerotic cardiovascular diseases. ${ }^{[16-19]}$ In the onset of diabetes, insulin resistance develops first. Causes of insulin resistance include reduction 
of glycogen synthesis in skeletal muscles by glucose transporter type-4 (GLUT 4), impaired insulin receptor binding or intracellular signal transduction, as well as the presence of high amounts of circulating free fatty acids. Patients with type 2 diabetes have insulin resistance and decreased $\beta$-cell function. Hyperglycemia causes islet cells to be constantly exposed to oxidative stress. Islet cells in the pancreas have a weaker antioxidant capacity. Long-term glucose toxicity and lipotoxicity cause $\beta$-cell failure.

Insulin resistance is related to the chronic increase of plasma glucose and triglycerides. ${ }^{[19]}$ The TyG index, which is an indicator of insulin resistance, is related to cardiovascular mortality and morbidity in patients with and without diabetes in many studies. ${ }^{[20]}$ The sensitivity and specificity of the TyG index for determining insulin resistance were $84.0 \%$ and $45.0 \%$, respectively, in the study reported by Simental-Mendia et al. ${ }^{[21]}$ Zhao et al. ${ }^{[22]}$ revealed that the TyG index could be a better predictor of cardiovascular risk than FPG or $\mathrm{HbA} 1 \mathrm{C}$ for patients with ACS.

In their study, da Silva et al. ${ }^{[23]}$ found that the TyG index could be used as a marker in determining the intensity of atherosclerosis in patients with symptomatic CAD. Mao et al. ${ }^{[24]}$ revealed that the TyG index could be a detached predictor of CAD severity as assessed by the SYNTAX score in patients with ACS. Luo et al. ${ }^{[25]}$ also observed that a TYG increase index could be a potent indicator of the worst prognosis in patients with acute ST-segment elevation myocardial infarction cured with percutaneous coronary intervention for one-year follow-up. In a cross-sectional study, patients with type 2 diabetes, but without a CAD history were found to have a higher TyG index related to an increased risk of significant coronary artery stenosis. ${ }^{[26]}$ In another study involving Korean adults, the TyG index was associated with the progression of coronary artery calcification. ${ }^{[27]}$

Recent studies have revealed that insulin resistance takes part in macrophage, endothelial, and vascular smooth muscle cell destruction, which contributes to plaque progression. ${ }^{[28]}$ Impaired glucose tolerance, increased insulin resistance increases oxidative stress in the long-term and damages vascular endothelial cells. Moreover, insulin resistance, hyperglycemia, and dyslipidemia increase plasminogen activator inhibitor-1 levels, resulting in reduced fibrinolytic activity and raised thrombotic events. ${ }^{[29,30]}$ Again, in several studies, insulin resistance causes both structural and functional deterioration such as increased intimamedia thickness, coronary artery calcification, and arterial stiffness in the vessel wall. ${ }^{[31]}$

In different studies, cardiovascular diseases and complications were found to be higher in diabetic patients. ${ }^{[32,33]}$ On the other hand, a higher level of TyG index can expedite atherosclerosis in patients with CABG that causes graft failure, as insulin resistance has a proinflammatory and procoagulatory effect, and $T y G$ index is related to endothelial dysfunction. In previous studies, increased insulin resistance levels were associated with the decreased circulation at the microvascular level. ${ }^{[34]}$

Insulin resistance was associated with an increased infarct area of single-photon emission computed tomography and myocardial perfusion imaging in a study that included non-diabetic patients with ST-segment elevation myocardial infarction. ${ }^{[35]}$ In our study, the TyG index was found to be higher in the group with poor CCC. This situation may have been the result of endothelial dysfunction as a result of the proinflammatory and procoagulatory effects of IR. ${ }^{[36,37]}$

The main limitation to this study is that it was conducted with a small group of patients in a single center. In addition, the retrospective nature of our study, including a small group of patients, reduces the power of the study. The findings may not cover other demographic groups. The TyG index of patients was calculated only once during hospitalization. Also, we could not reach the HbA1c results of all patients. Therefore, we did not include $\mathrm{HbA1c}$ results in the table as the statistical data. Calculating the changes in the TyG index during follow-up may be better in predicting the prognosis. Further multi-center, large-scale prospective studies are required to confirm our results.

In conclusion, high TyG index levels are related to poor collateral circulation in patients with CTO. A high TyG index is an important predictor of a low CCC grade. Based on these findings, TyG index can be a beneficial marker for prognosis in patients with type 2 diabetes and ACS undergoing percutaneous coronary intervention. For CTO interventions, these and similar markers can be a guide in the patient selection.

\section{Declaration of conflicting interests}

The authors declared no conflicts of interest with respect to the authorship and/or publication of this article. 


\section{Funding}

The authors received no financial support for the research and/or authorship of this article.

\section{REFERENCES}

1. Glovaci D, Fan W, Wong ND. Epidemiology of diabetes mellitus and cardiovascular disease. Curr Cardiol Rep 2019;21:21.

2. Sianos G, Barlis P, Di Mario C, Papafaklis MI, Büttner J, Galassi AR, et al; EuroCTO Club. European experience with the retrograde approach for the recanalisation of coronary artery chronic total occlusions. A report on behalf of the euroCTO club. EuroIntervention 2008;4:84-92.

3. Råmunddal $\mathrm{T}$, Hoebers LP, Henriques JP, Dworeck C, Angerås $\mathrm{O}$, Odenstedt $\mathrm{J}$, et al. Chronic total occlusions in Sweden--a report from the Swedish Coronary Angiography and Angioplasty Registry (SCAAR). PLoS One 2014;9:e103850.

4. Hakimzadeh N, Verberne HJ, Siebes M, Piek JJ. The future of collateral artery research. Curr Cardiol Rev 2014;10:73-86.

5. Du T, Yuan G, Zhang M, Zhou X, Sun X, Yu X. Clinical usefulness of lipid ratios, visceral adiposity indicators, and the triglycerides and glucose index as risk markers of insulin resistance. Cardiovasc Diabetol 2014;13:146.

6. Pala AA, Urcun YS. Relationship of triglycerideglucose index with chronic limb-threatening ischemia in lower extremity peripheral artery disease. Vascular 2021:17085381211018332.

7. Liu EQ, Weng YP, Zhou AM, Zeng CL. Association between triglyceride-glucose index and type 2 diabetes mellitus in the Japanese population: A secondary analysis of a retrospective cohort study. Biomed Res Int 2020;2020:2947067.

8. Low S, Khoo KCJ, Irwan B, Sum CF, Subramaniam T, Lim SC, et al. The role of triglyceride glucose index in development of type 2 diabetes mellitus. Diabetes Res Clin Pract 2018;143:43-9.

9. Ma X, Dong L, Shao Q, Cheng Y, Lv S, Sun Y, et al. Triglyceride glucose index for predicting cardiovascular outcomes after percutaneous coronary intervention in patients with type 2 diabetes mellitus and acute coronary syndrome. Cardiovasc Diabetol 2020;19:31.

10. Chiu TH, Tsai HJ, Chiou HC, Wu PY, Huang JC, Chen SC. A high triglyceride-glucose index is associated with left ventricular dysfunction and atherosclerosis. Int J Med Sci 2021;18:1051-7.

11. Lambrinoudaki I, Kazani MV, Armeni E, Georgiopoulos G, Tampakis K, Rizos D, et al. The TyG index as a marker of subclinical atherosclerosis and arterial stiffness in lean and overweight postmenopausal women. Heart Lung Circ 2018;27:716-24.

12. Alizargar J, Bai $\mathrm{CH}$. Comparison of carotid ultrasound indices and the triglyceride glucose index in hypertensive and normotensive community-dwelling individuals:
A case control study for evaluating atherosclerosis. Medicina (Kaunas) 2018;54:71.

13. Trifunovic D, Stankovic S, Sobic-Saranovic D, Marinkovic J, Petrovic M, Orlic D, et al. Acute insulin resistance in ST-segment elevation myocardial infarction in nondiabetic patients is associated with incomplete myocardial reperfusion and impaired coronary microcirculatory function. Cardiovasc Diabetol 2014;13:73.

14. Simental-Mendía LE, Rodríguez-Morán M, GuerreroRomero F. The product of fasting glucose and triglycerides as surrogate for identifying insulin resistance in apparently healthy subjects. Metab Syndr Relat Disord 2008;6:299-304.

15. Grossman C, Shemesh J, Koren-Morag N, Bornstein G, Ben-Zvi I, Grossman E. Serum uric acid is associated with coronary artery calcification. J Clin Hypertens (Greenwich) 2014;16:424-8.

16. Jin JL, Cao YX, Wu LG, You XD, Guo YL, Wu NQ, et al. Triglyceride glucose index for predicting cardiovascular outcomes in patients with coronary artery disease. J Thorac Dis 2018;10:6137-46.

17. Mesut E, Cihan A, Orhan G. Is it possible to predict the complexity of peripheral artery disease with atherogenic index? Vascular 2020;28:513-9.

18. Di Pino A, DeFronzo RA. Insulin resistance and atherosclerosis: Implications for insulin-sensitizing agents. Endocr Rev 2019;40:1447-67.

19. Ormazabal V, Nair S, Elfeky O, Aguayo C, Salomon C, Zuñiga FA. Association between insulin resistance and the development of cardiovascular disease. Cardiovasc Diabetol 2018;17:122.

20. Sánchez-Íñigo L, Navarro-González D, Fernández-Montero A, Pastrana-Delgado J, Martínez JA. The TyG index may predict the development of cardiovascular events. Eur J Clin Invest 2016;46:189-97.

21. Simental-Mendía LE, Rodríguez-Morán M, GuerreroRomero F. The product of fasting glucose and triglycerides as surrogate for identifying insulin resistance in apparently healthy subjects. Metab Syndr Relat Disord 2008;6:299-304.

22. Zhao Q, Zhang TY, Cheng YJ, Ma Y, Xu YK, Yang JQ, et al. Impacts of triglyceride-glucose index on prognosis of patients with type 2 diabetes mellitus and non-ST-segment elevation acute coronary syndrome: Results from an observational cohort study in China. Cardiovasc Diabetol 2020;19:108.

23. da Silva A, Caldas APS, Hermsdorff HHM, Bersch-Ferreira ÂC, Torreglosa CR, Weber B, et al. Triglyceride-glucose index is associated with symptomatic coronary artery disease in patients in secondary care. Cardiovasc Diabetol 2019;18:89.

24. Mao Q, Zhou D, Li Y, Wang Y, Xu SC, Zhao XH. The triglyceride-glucose index predicts coronary artery disease severity and cardiovascular outcomes in patients with non-ST-segment elevation acute coronary syndrome. Dis Markers 2019;2019:6891537.

25. Luo E, Wang D, Yan G, Qiao Y, Liu B, Hou J, et al. High triglyceride-glucose index is associated with poor prognosis in patients with acute ST-elevation myocardial infarction after percutaneous coronary intervention. Cardiovasc Diabetol 2019;18:150. 
26. Lee EY, Yang HK, Lee J, Kang B, Yang Y, Lee SH, et al. Triglyceride glucose index, a marker of insulin resistance, is associated with coronary artery stenosis in asymptomatic subjects with type 2 diabetes. Lipids Health Dis 2016;15:155.

27. Park K, Ahn CW, Lee SB, Kang S, Nam JS, Lee BK, et al. Elevated TyG index predicts progression of coronary artery calcification. Diabetes Care 2019;42:1569-73.

28. Reardon CA, Lingaraju A, Schoenfelt KQ, Zhou G, Cui C, Jacobs-El $\mathrm{H}$, et al. Obesity and insulin resistance promote atherosclerosis through an IFN $\gamma$-regulated macrophage protein network. Cell Rep 2018;23:3021-30.

29. Calles-Escandon J, Mirza SA, Sobel BE, Schneider DJ. Induction of hyperinsulinemia combined with hyperglycemia and hypertriglyceridemia increases plasminogen activator inhibitor 1 in blood in normal human subjects. Diabetes 1998;47:290-3.

30. Sobel BE. Insulin resistance and thrombosis: A cardiologist's view. Am J Cardiol 1999;84:37J-41J.

31. Adeva-Andany MM, Ameneiros-Rodríguez E, FernándezFernández C, Domínguez-Montero A, Funcasta-Calderón $\mathrm{R}$. Insulin resistance is associated with subclinical vascular disease in humans. World J Diabetes 2019;10:63-77.

32. Yeşilkaya $N$, Gökalp $O$, İner $H$, Beşir $Y$, Durmaz $H$, Gökkurt Y, et al. Effects of systemic inflammatory response on coronary artery bypass grafting. Cardiovascular Surgery and Interventions 2020;7:8-13.

33. Çakır M, Çakır H, Dönmez K, Karaagaç E, Yürekli İ, Öngel K, et al. Status of preventive services against coronary risk factors in primary healthcare for patients undergoing coronary artery bypass grafting. Cardiovasc Surg Int 2021;8:1-7.

34. Alur İ, Pekel G, Durna F, Bahar A, Alihanoğlu Yİ, Gökşin İ. Evaluation of retinal vessel caliber, choroidal thickness, and ocular perfusion pressure in patients with low cardiac ejection fraction. Cardiovasc Surg Int 2019;6:1-6.

35. Trifunovic D, Stankovic S, Sobic-Saranovic D, Marinkovic J, Petrovic M, Orlic D, et al. Acute insulin resistance in ST-segment elevation myocardial infarction in nondiabetic patients is associated with incomplete myocardial reperfusion and impaired coronary microcirculatory function. Cardiovasc Diabetol 2014;13:73.

36. Engin $M$, Güvenç O. Investigation of the predictive values of triglyceride/HDL cholesterol ratio and whole blood viscosity with regard to severe peripheral or carotid artery disease in patients scheduled for coronary bypass. Heart Surg Forum 2020;23:E310-E314.

37. Nakagomi A, Sunami Y, Kawasaki Y, Fujisawa T, Kobayashi Y. Sex difference in the association between surrogate markers of insulin resistance and arterial stiffness. J Diabetes Complications 2020;34:107442. 